\title{
Construção de um índice global de impacto para análise ambiental comparativa aplicada à adequação de empreendimentos irregulares
}

\author{
Construction of an impact global index for comparative \\ environmental analysis applied to the settlement of irregular activities
}

\begin{abstract}
Adriano Bressane', Fábio Augusto Gomes Vieira Reis², Lucilia do Carmo Giordano², José Arnaldo Frutuoso Roveda ${ }^{3}$, Sandra Regina Monteiro Masalskiene Roveda ${ }^{3}$, Antonio Cesar Germano Martins ${ }^{4}$, Patricia Satie Mochizuki ${ }^{5}$
\end{abstract}

\section{RESUMO}

Por muito tempo o exercício de atividades econômicas ocorreu na ausência de normas reguladoras. Com a normatização, muitos empreendimentos enquadraram-se em situação de irregularidade, sobretudo por ocuparem obras situadas em áreas especialmente protegidas. Nessa condição, a manutenção ou o desfazimento dessas obras deve se basear em uma análise comparativa dos impactos decorrentes de ambas as alternativas, entretanto a falta de critérios e de padronização nos procedimentos tem prejudicado o adequado tratamento e respectiva tomada de decisões. Assim, o presente trabalho teve como objetivo apresentar uma proposta metodológica para análise ambiental comparativa aplicadaà adequação deempreendimentos irregulares. Paratanto,foi construído um índice global de impacto, mediante revisão bibliográfica, que possibilitou identificar os parâmetros envolvidos e o seu equacionamento com o uso de matrizes de interação. O método foi aplicado em um empreendimento que realizou intervenção em uma Área dePreservação Permanente, no limite urbano consolidado sem prévia autorização do órgão licenciador. Como resultado, alcançou-se um índice capaz de subsidiar tomadas de decisão quanto à manutenção ou ao desfazimento de empreendimentos irregulares, levando em consideração atributos como duração, extensão e intensidade no equacionamento da magnitude do impacto, bem como os parâmetros de acumulação, reversibilidade e sensibilidade para avaliar sua importância. Embora o método proposto não elimine a subjetividade da avaliação ambiental, contribui para sua padronização por meio de um procedimento lógico e organizado que propicia comparação fundamentada em parâmetros quantitativos, de maneira especial para empreendimentos de menor porte, em geral avaliados de acordo com atributos unicamente qualitativos.

Palavras-chave: análise comparativa; impacto ambiental; regularização ambiental.

\begin{abstract}
For a long time, economic activities occurred in the lack of legal rules. Due to advancement of legislation, many activities became in situation of irregularity, mainly by occupying buildings in protected areas. In this condition, the maintenance or destruction of these buildings should be based on an impact comparative analysis of both alternatives. However, a lack of criteria and standardization of procedures has undermined the proper treatment and decision making. Thus, the purpose of this paper was to introduce a methodological proposal for comparative environmental analysis applied to the settlement of irregular activities. For that, an impact global index was constructed, by means of literature review to identify the parameters involved, as well as to its formulation with use of interaction matrices. The method was applied for assessing an activity that made intervention in permanent preservation area, within consolidated urban area without authorization of competent agency. As a result, an index able to support makingdecision about maintenance or destruction of irregular buildings was obtained, considering attributes as duration, extension and intensity in the formulation of magnitude of the impact, as well as the parameters of accumulation, reversibility and sensibility to evaluate its importance. Although the proposed method does not eliminate the subjectivity of the environmental assessment, it contributes to standardization through a logical and organized procedure that allows a comparison based on quantitative parameters, especially for smaller projects, usually assessed only according to qualitative attributes.
\end{abstract}

Keywords: comparative analysis; environmental impact; environmental regularization.

$\square$

'Doutorado em Ciências Ambientais pela Universidade Estadual Paulista (UNESP) - Sorocaba (SP), Brasil.

2Doutorado em Geociências e Meio Ambiente pela UNESP - Rio Claro (SP), Brasil.

${ }^{3}$ Doutorado em Matemática pela Universidade de Brasilia (UnB) - Brasília (DF), Brasil.

${ }^{4}$ Doutorado em Engenharia Elétrica pela Universidade de São Paulo (USP) - São Paulo (SP), Brasil.

${ }^{5}$ Especialista Ambiental pela Secretaria do Meio Ambiente do Estado de São Paulo (SMA) - São Paulo (SP). Brasil.

Endereço para correspondência: Adriano Bressane - Avenida Três de Março, 511 - Alto da Boa - 18087-180 - Sorocaba (SP), Brasil - E-mail: adriano.bressane@posgrad.sorocaba.unesp.br Recebido: 11/12/14 - Aceito: 17/05/16 - Reg. ABES: 140136 


\section{INTRODUÇÃO}

Com a finalidade de sintetizar informações sobre uma determinada área ou fenômeno, frequentemente são empregados indicadores e índices como instrumentos de avaliação, entre os quais estão as avaliações de impacto ambiental. Enquanto um indicador pode ser composto por um único parâmetro ou agregado de informações (MITCHELL, 1996), o índice consiste no valor que resulta de um procedimento de cálculo em que se utilizam indicadores como variáveis (SICHE et al., 2007). Ou seja, pode-se dizer ainda que um índice é um indicador de alta categoria (KHANNA, 2000).

O processo de elaboração de índices intensificou-se a partir da década de 1970, pela necessidade de subsídios à gestão ambiental (SANTOS, 2004). Desde então, diversas foram as criações desse instrumento. Entre outros, podem ser destacados emergy sustainability index (BROWN \& ULGIATI, 1997), environmental sustainability index (SAMUEL-JOHNSON \& ESTY, 2000) e ecological footprint (WACKERNAGEL \& REES, 1996).

Pelo estudo desses exemplos, constata-se que os índices são dinâmicos e estão com frequência sendo aperfeiçoados (o que também se pretende realizar neste trabalho), por meio de matrizes de interação, para apoiar a adequação de atividades irregulares.

As matrizes de interação estão entre os métodos mais comumente empregados em estudos de impacto ambiental, com destaque aos métodos de Fischer e Davies (1973) e Leopold et al. (1971), os quais têm sofrido numerosas adaptações visando à sua adaptação às particularidades de cada caso, bem como as exigências da legislação (TOMMASI, 1993; SÁNCHEZ, 2006). Originalmente, a matriz de Leopold et al. (1971) consistia em uma listagem bidimensional organizada em um quadro em que nas colunas estão ações do projeto e nas linhas os fatores ambientais, de modo que nas células formadas pelo cruzamento das linhas com as colunas são assinalados com o sinal de (+) os impactos positivos e com (-) os negativos, além da atribuição de valores de 1 a 10 para os parâmetros de importância e magnitude - escala alterada para de 1 a 5 por Fischer e Davies (1973).

De modo geral, os riscos de degradação tornaram os estudos de impacto ambiental uma exigência legal para atividades potencialmente poluidoras, objetivando a aplicação de medidas de controle, bem como o subsídio de decisões sobre a viabilidade de empreendimentos (COMISSÃO MUNDIAL SOBRE O MEIO AMBIENTE E DESENVOLVIMENTO, 1991).

No Brasil, a legislação define impacto ambiental como:

qualquer alteração das propriedades físicas, químicas e biológicas do meio ambiente, causada por qualquer forma de matéria ou energia resultante das atividades humanas que, direta ou indiretamente, afetam: a) a saúde, a segurança e o bem-estar da população; b) as atividades sociais e econômicas; c) a biota; d) as condições estéticas e sanitárias do meio ambiente; e) e a qualidade dos recursos ambientais (BRASIL, 1986).

A mesma normativa, a Resolução do Conselho Nacional do Meio Ambiente (Conama) n. ${ }^{\circ}$, de 1986, exige a análise das alternativas de projeto, sobretudo da previsão de magnitudes, e a interpretação da importância dos prováveis impactos delas decorrentes, discriminando: os positivos e negativos (benéficos e adversos); os diretos e indiretos; os imediatos e a médio e longo prazos; os temporários e permanentes; o grau de reversibilidade; as propriedades cumulativas e sinérgicas; e a distribuição dos ônus e benefícios sociais.

Embora essa exigência seja uma condição prévia à implantação de atividades modificadoras do meio ambiente, não são raros os casos em que tais atividades se encontram irregularmente em operação sem a licença ambiental necessária e, em muitos deles, em áreas especialmente protegidas, como as de preservação permanente (APP), em geral associadas a atividades ou empreendimentos de pequeno e médio portes.

Nessas situações, a regularização deveria implicar o desfazimento da atividade e a recuperação da área a sua condição anterior. Contudo, segundo o Decreto federal n. ${ }^{\circ} 6.514$ de 2008 :

Não será aplicada a penalidade de demolição quando, mediante laudo técnico, for comprovado que o desfazimento poderá trazer piores impactos ambientais que sua manutenção, caso em que a autoridade ambiental, mediante decisão fundamentada, deverá, sem prejuízo das demais sanções cabíveis, impor as medidas necessárias à cessação e mitigação do dano ambiental, observada a legislação em vigor (BRASIL, 2008).

Logo, o atendimento de tais diretrizes requer um procedimento metodológico que proporcione satisfatória clareza na estrutura de integração dos dados e aplicação dos critérios na avaliação, pois se trata de uma decisão quanto ao tratamento dos casos de atividades irregulares, o que representa fator de alta relevância para a gestão da qualidade ambiental, sobretudo em APPs.

Todavia, a falta de critérios e de padronização nos procedimentos de análise tem prejudicado o adequado tratamento e respectiva tomada de decisões. Nesse contexto, os objetivos deste trabalho foram o desenvolvimento e a proposição de um método de avaliação ambiental comparativa como suporte à revisão de atividades irregulares, bem como sua aplicação a um caso de obra situada em APP de um corpo hídrico, visando orientar decisões relacionadas a sua manutenção ou a seu desfazimento.

\section{METODOLOGIA}

Por se tratar de uma pesquisa de base bibliográfica, para o desenvolvimento do estudo, os principais materiais utilizados corresponderam 
a obras da literatura técnica e normas da legislação aplicável, da análise de obras gerais para as especializadas, conforme recomendações de Ruiz (1996). Assim, com base em tais referências, foram definidas as variáveis, bem como a forma de sua integração.

Logo, o procedimento metodológico gerado para proporcionar uma avaliação ambiental comparativa como suporte à decisão em processos de regularização foi desenvolvido mediante a construção de um índice global de impacto (IGI).

Os métodos atuais envolvendo o uso de tais matrizes, tal como em Leopold et al. (1971) e Fisher e Davies (1973), não geram valor final único, dificultando análises ambientais comparativas de forma direta e sistemática, motivação pela qual se desenvolveu a proposta de um índice global aplicado a essa finalidade.

\section{RESULTADOS E DISCUSSÃO}

\section{Construção do índice global de impacto}

\section{Identificação dos parâmetros e alinhamento conceitual}

Embora exista determinação normativa quanto à previsão de magnitude e interpretação da importância dos impactos (BRASIL, 1986), não há orientação sobre o entendimento dessas variáveis e das demais (SÁNCHEZ, 2006), muito menos padronização de equacionamento entre elas (OLIVEIRA \& MOURA, 2009).

Para tanto, a construção do IGI baseou-se na sistematização de operações matemáticas abrangendo ponderações de magnitude $(\mu)$ e importância $(i)$ cujo equacionamento indica a significância $(\sigma)$ do impacto das alternativas de projeto avaliadas, neste estudo representadas pelos cenários de manutenção e de desfazimento da obra.
Nesse sentido, os parâmetros foram definidos em atendimento à legislação aplicável (BRASIL, 1986), sendo a conotação atribuída de acordo com a fundamentação alcançada com base na revisão bibliográfica, resultando no alinhamento conceitual proposto no Quadro 1.

Assim, propõe-se que os parâmetros de duração, extensão e intensidade correspondam a ponderadores de quantificação da magnitude dos impactos. Da mesma forma, a acumulação, a reversibilidade e a sensibilidade seriam aplicadas como ponderadores para qualificação de sua importância. Para isso, tais ponderadores seriam classificados segundo os modificadores exibidos no Quadro 2.

Logo, enquanto os ponderadores $\left(P_{j}\right)$ constituem parâmetros que quantificam a magnitude $(\mu)$ do impacto e qualificam sua importância $(i)$, os modificadores $\left(m_{k j}\right)$ correspondem às classes que caracterizam a condição avaliada para cada um desses parâmetros, em conformidade com a que são atribuídos os pesos $\left(w_{k j}\right)$.

A escala de valores atribuída a tais pesos foi proposta visando estabelecer um intervalo de variação conveniente para o IGI entre -1 e 1, e assim facilitar a avaliação comparativa entre os cenários analisados.

Conforme o equacionamento apresentado adiante, o IGI resulta de um procedimento de cálculo em que inicialmente valores de magnitude e importância de cada impacto são obtidos agregando pela soma o valor dos seus ponderadores. Na sequência, a significância do impacto é dada pelo produto de sua magnitude pela importância.

Para isso, na valoração dos numeradores de cada peso foi adotada uma escala arbitrária $(0,1,2,3)$, na qual os limites 0 e 3 expressam, respectivamente, o menor e o maior peso relativo à condição avaliada pelos ponderadores de cada parâmetro. Então, para alcançar os limiares inferior (-1) e superior (1) do IGI, aos pesos $\left(w_{k j}\right)$ foi atribuído como denominador o valor equivalente a soma dos numeradores mais elevados em cada modificador, o que totaliza 9 (para a soma dos dividendos de $w_{31}, w_{32}$ e $w_{33}$ no caso da magnitude, por exemplo).

Quadro 1 - Alinhamento conceitual proposto para aplicação do índice global de impacto.

\begin{tabular}{|c|l|}
\hline Parâmetro & \multicolumn{1}{c|}{ Alinhamento conceitual } \\
\hline Magnitude & $\begin{array}{l}\text { Característica do impacto relacionada à quantificação dos efeitos sobre o meio ambiente, em termos de duração, } \\
\text { extensão e intensidade }\end{array}$ \\
\hline Duração & Quantificação da amplitude temporal do impacto; tempo de permanência \\
\hline Extensão & Quantificação da abrangência espacial do impacto; território atingido \\
\hline Intensidade & Quantificação do nível de alteração da característica ambiental; grau de modificação \\
\hline Importância & $\begin{array}{l}\text { Característica do impacto relacionada à qualificação dos efeitos sobre o meio ambiente, em termos de acumulação, } \\
\text { reversibilidade e sensibilidade }\end{array}$ \\
\hline Acumulação & Qualificação quanto à possibilidade de melhoria ou agravamento das alteraçães \\
\hline Reversibilidade & Qualificação quanto à capacidade de retorno a uma situação de equilíbrio ambiental \\
\hline Sensibilidade & Qualificação quanto à tolerância às alterações considerando a condição atual \\
\hline Significância & Síntese da análise de impactos ambientais pela integração dos parâmetros quantitativos e qualitativos \\
\hline
\end{tabular}




\section{Formulação do índice global de impacto}

Considerando o intervalo de variação do IGI entre -1 e 1 e que os impactos podem ter natureza positiva (benéfica) ou negativa (adversa), os limiares inferior (-1) e superior (1) representam o pior e o melhor cenário possível, respectivamente. Para isso, propõe-se (Equação 1):

igi $=\frac{1}{n} \sum_{i=1}^{n} \sigma_{i}$

\section{Em que:}

$i g i$ = índice global de impacto para a alternativa avaliada;

$n=$ número total de impactos;

$i=(1,2, \ldots, n)$;

$\sigma_{i}=$ significância do impacto $i$, dada por (Equação 2):

$\sigma_{i}=\mu_{i} \cdot r_{i}$

Em que:

$\mu_{i}=$ magnitude do impacto $i$;

$\mathbf{l}_{i}=$ importância do impacto $i$.

Quadro2-Proposta de modificadores e respectivos pesos para ponderação.

\begin{tabular}{|c|c|c|c|}
\hline Atributo & Ponderador $(P)$ & Modificador $\left(m_{k j}\right)$ & $\operatorname{Peso}\left(w_{k j}\right)$ \\
\hline \multirow{9}{*}{ Magnitude $(\mu)$} & \multirow{3}{*}{ Duração $\left(P_{1}\right)$} & Curta a média $\left(m_{11}\right)$ & $1 / 9\left(m_{11}\right)$ \\
\hline & & Longo prazo $\left(m_{21}\right)$ & $2 / 9\left(w_{21}\right)$ \\
\hline & & Permanente $\left(m_{31}\right)$ & $3 / 9\left(w_{31}\right)$ \\
\hline & \multirow{3}{*}{ Extensão $\left(P_{2}\right)$} & Pontual $\left(m_{12}\right)$ & $1 / 9\left(w_{12}\right)$ \\
\hline & & Local $\left(m_{22}\right)$ & $2 / 9\left(w_{22}\right)$ \\
\hline & & Regional $\left(m_{32}\right)$ & $3 / 9\left(w_{32}\right)$ \\
\hline & \multirow{3}{*}{ Intensidade $\left(P_{3}\right)$} & Baixa $\left(m_{13}\right)$ & $1 / 9\left(w_{13}\right)$ \\
\hline & & Média $\left(m_{23}\right)$ & $2 / 9\left(w_{23}\right)$ \\
\hline & & Alta $\left(m_{33}\right)$ & $3 / 9\left(w_{33}\right)$ \\
\hline \multirow{9}{*}{ Importância (i) } & \multirow{3}{*}{ Acumulação $\left(P_{4}\right)$} & Ausente $\left(m_{44}\right)$ & $\mathrm{O}\left(w_{44}\right)$ \\
\hline & & Incerta $\left(m_{54}\right)$ & $2 / 9\left(w_{54}\right)$ \\
\hline & & Presente $\left(m_{64}\right)$ & $3 / 9\left(w_{64}\right)$ \\
\hline & \multirow{3}{*}{ Reversibilidade $\left(P_{5}\right)$} & $\operatorname{Sim}\left(m_{45}\right)$ & $1 / 9\left(w_{45}\right)$ \\
\hline & & Talvez $\left(m_{55}\right)$ & $2 / 9\left(W_{55}\right)$ \\
\hline & & Não $\left(m_{65}\right)$ & $3 / 9\left(w_{65}\right)$ \\
\hline & \multirow{3}{*}{ Sensibilidade $\left(P_{6}\right)$} & $\operatorname{Nula}\left(m_{46}\right)$ & $\mathrm{O}\left(w_{46}\right)$ \\
\hline & & Parcial $\left(m_{56}\right)$ & $2 / 9\left(w_{56}\right)$ \\
\hline & & Total $\left(m_{66}\right)$ & $3 / 9\left(w_{66}\right)$ \\
\hline
\end{tabular}

Tanto a magnitude do impacto quanto a importância do impacto são calculados por meio da Equação 3:

$\mu_{i}=\sum_{j=1}^{3}\left(p_{j}\right)_{i}$

e pela Equação 4

$\mathrm{u}_{i}=\sum_{j=4}^{6}\left(p_{j}\right)_{i}$

Tendo $\left(p_{j}\right)_{i}$ como o valor do ponderador $j$ para o impacto $i$, calculado para magnitude pela Equação 5:

$\left(p_{j}\right)_{i}=\sum_{k=1}^{3}\left(w_{k j} \cdot m_{k j}\right)_{i}$

Para importância, pela Equação 6:

$\left(p_{j}\right)_{i}=\sum_{k=4}^{6}\left(w_{k j} \cdot m_{k j}\right)_{i}$

Em que:

$\left(w_{k j}\right)_{i}=$ peso do modificador $k$ pertencente ao ponderador $j$ para o impacto $i$, conforme valores propostos no Quadro 2;

$\left(m_{k j}\right)_{i}=$ valor do modificador $k$ pertencente ao ponderador $j$ para o impacto $i$, o qual representa a condição avaliada para o impacto segundo cada ponderador, dado por meio da Equação 7:

$\left(m_{k j}\right) i=\left\{\begin{array}{l}1, \text { se pertence a condição avaliada } \\ -1, \text { se a condição está relacionada e o impacto é prejudicial } \\ 0, \text { caso contrário }\end{array}\right.$

Com base nessas definições dadas pelas Equações 8 e 9:

$\mathbf{M}=\left[\mu_{1} \mu_{2} \ldots \mu_{n}\right]$

e

$\mathbf{I}=\left[\begin{array}{llll}\mathbf{l}_{1} & \mathrm{l}_{2} & \ldots & \mathrm{l}_{n}\end{array}\right]$

$\mathrm{Na}$ qual:

$\mathbf{M}=$ vetor de magnitude dos impactos;

$\mathbf{I}=$ vetor de importância dos impactos.

Têm-se (Equação 10):

igi $=\frac{1}{n} \sum_{i=1}^{n} \sigma_{i}=\frac{1}{n} \sum_{i=1}^{n} \mathrm{M}_{i} \mathrm{I}_{i}=\frac{1}{n}\left(\mathbf{M} . \mathbf{I}^{\mathrm{T}}\right)$

Considerando uma análise comparativa entre duas ou mais alternativas de projeto, tem-se a generalização da fórmula (Equação 10), dada por meio da Equação 11: 
$i g i_{\lambda \tau}=\left(\frac{1}{n} \sum_{i=1}^{n}\left(\sigma_{i}\right)_{\lambda \tau}\right)=\left(\frac{1}{n} \sum_{i=1}^{\mathrm{n}}\left(\mathbf{M}_{i \lambda \tau} . \mathbf{I}_{i \lambda \tau}\right)\right)$

Composta por:

$i g i_{\lambda \tau}=$ índice global de impacto da alternativa de projeto $\left(A_{\lambda \tau}\right)$, composta pela tecnologia $\tau$ associada à localização $\lambda$;

$\lambda=(1,2, \ldots, l)$, em que $l$ é o número total de alternativas locacionais avaliadas;

$\tau=(1,2, \ldots, t)$, em que $t$ é o número total de alternativas tecnológicas avaliadas.

Logo, para efeito de organização dos dados, temos (Equação 12):

$\boldsymbol{I} \boldsymbol{G} \boldsymbol{I}=\delta_{\lambda \tau}\left(\frac{1}{n}\left(\mathbf{M}_{\lambda \tau} \cdot\left[\mathbf{I}_{\lambda \tau}\right]^{\mathrm{T}}\right)\right)=\left[i g i_{11} i g i_{12} \ldots i g i_{l t}\right]$

Sendo:

IGI = matriz composta pelos IGIs de cada cenário avaliado;

$\delta_{\lambda \tau}=$ distribuição delta de Dirac, dada por meio da Equação 13:

$\delta_{\lambda \tau}=\left\{\begin{array}{l}1 s e \lambda=\tau \\ 0 \text { caso contrário }\end{array}\right.$

Desse modo, a melhor alternativa de projeto $A_{\lambda^{\prime} \tau^{\prime}}$ entre as avaliadas comparativamente será aquela com maior igi (impactos mais positivos ou menos negativos), dada pela Equação 14:

$A_{\lambda^{\prime} \tau^{\prime}}=\max (\boldsymbol{I G I})$

Em que:

$\lambda^{\prime}=$ melhor alternativa locacional (menos sensível aos impactos por $\tau^{\prime}$ ); $\tau^{\prime}=$ melhor alternativa tecnológica (menos severa na geração de impactos sobre $\left.\lambda^{\prime}\right)$.

\section{Aplicação do índice para apoiar a adequação da obra irregular em estudo}

\section{Estudos de base}

Para fundamentar as ponderações dos parâmetros de magnitude e importância, são necessários estudos de base (SÁNCHEZ, 2006) quanto à caracterização dos atributos de localização e de alteração provocada pela manutenção e pelos impactos previstos para o cenário de desfazimento da obra.

No estudo de caso em análise, avaliaram-se as alternativas de projeto para uma obra de estacionamento de um estabelecimento comercial, implantada sem prévia autorização em APP, situada em determinada localidade do município de Guarulhos (SP). Na referida área, foram então avaliados os atributos de localização a seguir apresentados.
A geologia sob o empreendimento pertence à Formação São Paulo, constituída predominantemente de argilas, siltes e areias argilosas. Localmente, é composta por sedimentos aluvionares fluviais (COUTINHO, 1984). Logo, mesmo na ausência de lineamentos estruturais (falhas), tais características conferem à área baixa estabilidade geológica.

A pedologia local corresponde a solos de alteração em rochas cristalinas da classe dos latossolos vermelho amarelo (porção leste), de textura siltosa, baixa suscetibilidade à erosão e baixa condutividade hidráulica; e solos aluviais da classe Gley Hidromórfico, com textura argilossiltosa, muito baixa condutividade hidráulica e muito baixa suscetibilidade à erosão (ANDRADE, 1999).

De acordo com Pires Neto (2004), a geomorfologia da área corresponde ao Planalto Atlântico, subzona Colinas de São Paulo, cujas formas de relevo sob o empreendimento consistem em morrotes paralelos (porção leste) e planície fluvial (porção oeste).

O Córrego dos Cubas (afluente do Rio Tietê), cuja APP sofreu intervenção pela obra em estudo, pertence à bacia do Alto Tietê, sub-bacia do Canal de Circunvalação, no entorno da qual se enquadram áreas com risco de inundação variável de baixo a médio-alto. (GUARULHOS, 2004b).

O padrão de drenagem possui canais de média a baixa densidade, com padrão subparalelo à dendrítico, com vales fechados e interflúvios sem orientação e área inferior a $1 \mathrm{~km}^{2}$ (IPT, 1981).

Referente à quantidade hídrica, constata-se que o Córrego dos Cubas possui pequeno caudal, com largura média de $2 \mathrm{~m}$, e que no entorno da área está parcialmente canalizado a montante do empreendimento e completamente canalizado a partir de $500 \mathrm{~m}$ a jusante. Quanto à qualidade das águas, o recebimento de esgoto sanitário não tratado nos últimos anos provocou elevado grau de poluição (aspectos físico-químicos fortemente alterados) e contaminação por coliformes fecais.

Conforme a base de dados do Instituto Florestal (IF, 2005), a composição florística regional pertence à vegetação secundária da floresta ombrófila submontana, no entanto a forte urbanização no entorno da área do empreendimento suprimiu significativamente os fragmentos florestais ao sul do empreendimento.

No tocante ao incremento de conectividade da vegetação, o local em que se insere o empreendimento pertence à Classe 2, a qual pode ser interpretada como baixo grau de prioridade (SÃO PAULO, 2007).

Resultados do levantamento da Secretaria de Meio Ambiente de Guarulhos (GUARULHOS ONLINE, 2010) mostram que a fauna silvestre presente no município totaliza cerca de 500 espécies concentradas nas áreas conservadas, entre as quais: 95 são de invertebrados; três de peixes; 28 de anfíbios; 55 de répteis; 63 de mamíferos; e 261 de aves. 
Por sua vez, na área do empreendimento e seu entorno, por conta do expressivo avanço do processo de urbanização e tamponamento de cursos hídricos, mesmo antes do início das obras em análise, já se constatava consolidado o afugentamento da macrofauna terrestre - tanto pela supressão do hábitat quanto pelo controle de zoonoses - , bem como a inexistência de espécies aquáticas no córrego interceptado.

Segundo o zoneamento municipal, o local do empreendimento pertence à zona de uso misto, componente da macrozona de urbanização consolidada, a qual se caracteriza por áreas dotadas de média ou boa infraestrutura urbana com vocação para o adensamento populacional, alta incidência de usos habitacionais, comércio e prestação de serviços (GUARULHOS, 2004a).

Em conformidade com a vocação prevista pela legislação municipal, as áreas no entorno do empreendimento constituíram cenário de progressiva ocupação, ao longo da qual propriedades rurais com estradas de terra (1977) deram lugar a loteamentos urbanos (1986), ao crescente adensamento populacional (1998) e à pavimentação das vias de circulação (2004), conforme análise temporal do uso do solo realizado por fotografias aéreas.

Anteriormente à implantação do empreendimento na área, a urbanização no seu entorno já havia concretizado significativas transformações no ambiente natural por supressões da vegetação, movimentações de terra, impermeabilizações do solo, edificações em APP, assoreamento e despejos de esgoto sanitário em cursos hídricos, entre outras. Mais tarde, galpões existentes na área foram demolidos (2006), e o córrego foi transposto e canalizado (2009).

\section{Identificação dos impactos ambientais $\left(i_{\mathrm{n}}\right)$}

\section{Poluição dos corpos hídricos (i):}

- manutenção da obra: não provoca ou agrava geração de qualquer matéria ou energia que resulte em alteração na qualidade da água; a pavimentação do solo e as medidas de drenagem adotadas disciplinam o deflúvio; há prevenção quanto à ocorrência de transporte e sedimentação de materiais para o curso hídrico; há prejuízos à recarga do lençol, mas o solo local já possui baixa condutividade hidráulica natural;

- desfazimento da obra: permitiria a revegetação; proporcionaria a retenção ciliar de poluentes; acarretaria a produção de matéria (entulhos) que poderia resultar em alteração temporária na qualidade (turbidez) e quantidade (assoreamento) da água.

\section{Estabilidade geológica (i $)$ :}

- manutenção da obra: não provoca ou agrava geração de qualquer pressão significativa sobre a estrutura geológica que resulte em riscos à sua estabilidade; minimização de tal risco pela instalação de gabiões ao longo do canal e pela pavimentação do solo no entorno, pois a estabilidade das condições naturais é baixa.

- desfazimento da obra: permitiria a revegetação, que, por sua vez, proporcionaria a estabilização dos taludes marginais. No entanto, durante o período de plantio e regeneração, haverá fragilização dos taludes marginais, pela exposição do substrato que poderia resultar em erosão.

\section{Degradação do solo (i $\boldsymbol{i}_{3}$ :}

- manutenção da obra: não provoca ou agrava a ocorrência de processos erosivos do solo. Ao contrário, a sua pavimentação protege-o, pois anula as forças erosivas do impacto da chuva e do escoamento pluvial;

- desfazimento da obra: permitiria a revegetação, que, por sua vez, minimizaria as forças erosivas da chuva e do escoamento pluvial. Todavia, durante o período de desconstrução haveria exposição do pavimento desprotegido, que poderia resultar em perda de solo.

\section{Perda de biodiversidade $\left(\boldsymbol{i}_{4}\right)$ :}

- manutenção da obra: provoca o impedimento da regeneração florestal ciliar, desfavorecendo a biota, bem como provoca o seu afugentamento no entorno da área, mas não há evidências de espécimes endêmicas e/ou ameaçadas de extinção no local. Contudo há ainda que se considerar que a presença de grande proporção de espécies da fauna silvestre em área urbana consolidada representa riscos à saúde e segurança da população, seja pela veiculação de doenças, seja por acidentes diversos;

- desfazimento da obra: permitiria a revegetação florestal, que, por sua vez, proporcionaria a recuperação do hábitat de espécies silvestres da fauna e flora. No entanto não se pode desprezar que o efeito de borda provocado pela intensa urbanização no entorno, bem como o tamponamento do canal a jusante, prejudicaria fortemente as condições favoráveis ao seu estabelecimento. Ademais, reitera-se que a presença na área urbana de espécies silvestres, entre as quais se podem citar capivaras, morcegos, roedores, cobras (répteis em geral), representaria sério risco à população.

\section{Comprometimento do fluxo gênico de fauna e flora ( $\boldsymbol{i}_{5}$ :}

- manutenção da obra: provoca o impedimento da regeneração florestal ciliar, cerceando o fluxo de espécies silvestres da flora e fauna. Entretanto, além das considerações anteriormente expostas, a área em questão não constitui entremeio (corredor ecológico) a fragmentos florestais no seu entorno, visto que estes ocorrem a moderadas distâncias somente ao norte do local;

- desfazimento da obra: permitiria a revegetação florestal, que, por sua vez, proporcionaria o fluxo gênico de fauna e flora, entretanto 
não se pode desprezar que o efeito de borda provocado pela intensa urbanização no entorno prejudicaria as condições favoráveis ao seu estabelecimento. Ademais, reiteram-se os riscos relacionados à presença de espécies silvestres na área densamente urbanizada.

\section{Poluição visual ( $\left.\boldsymbol{i}_{6}\right)$ :}

- manutenção da obra: impossibilita a renaturalização da paisagem local, no entanto não se pode desconsiderar que a artificialização relativa à transposição e à canalização do curso hídrico visa adequá-la à vocação da área, em conformidade ao planejamento urbano vigente. A manutenção do pavimento impermeável do estacionamento não impossibilita que seja executado um projeto paisagístico por arborização com espécies nativas em covas entre as vagas que o compõem;

- desfazimento da obra: permitiria a revegetação, que, por sua vez, proporcionaria a renaturalização da paisagem, mas com reduzida melhoria por conta do bom estado de conservação atual da área, que melhor se ajusta à vocação local da ocupação no entorno e pode ter efeitos adversos mitigados.

\section{Comprometimento das condições}

sanitárias, de saúde e bem-estar (i $)$ :

- manutenção da obra: proporciona a conservação das condições sanitárias por ela proporcionadas, entre as quais se destacam a prevenção quanto à ocorrência de inundações a jusante (pelo aumento da vazão) e a recuperação da qualidade hídrica, que antes recebia despejos sanitários sem tratamento a montante;

- desfazimento da obra: comprometeria a conservação das melhorias sanitárias por ela proporcionadas (anteriormente descritas).

\section{Comprometimento da viabilidade econômica ( $\left.\boldsymbol{i}_{8}\right)$ :}

- manutenção da obra: proporciona melhores condições de atendimento aos clientes do estabelecimento comercial (maior número de vagas) e, consequentemente, permite atender a uma demanda maior, gera melhor expectativa de prosperidade financeira (satisfação do empreendedor quanto à segurança do investimento), mais contratação de mão de obra (geração de emprego e renda), indução do desenvolvimento econômico e da infraestrutura local (estímulo a outros investidores, fomentando novos empreendimentos e respectiva maior de arrecadação tributária);

- desfazimento da obra: comprometeria a capacidade de atendimento a um maior número de clientes do estabelecimento comercial, reduzindo sua expectativa de prosperidade e, respectivamente, a segurança do investidor na contratação de mais mão de obra, induzindo os demais efeitos colaterais antes descritos, que, em síntese, criam condições adversas à viabilidade econômica, além de gerar maior pressão sobre o sistema viário local, no que tange ao estacionamento de veículos na via pública.

\section{Análise dos impactos e avaliação}

\section{comparativa das alternativas}

No caso da análise em estudo, isto é, adequação de obra irregular, as alternativas de projeto $\left(A_{\lambda \tau}\right)$ avaliadas correspondem a dois cenários equivalentes à manutenção $(m)$ e ao desfazimento $(\mathrm{d})$ da obra.

Logo, como em ambos os cenários a localização $(\lambda)$ é a mesma (obra existente), as alternativas podem ser identificadas simplesmente por $A_{m}$ para o cenário de manutenção $(\lambda 1=m)$ e $A_{d}$ para o cenário de desfazimento $(\lambda 2=d)$.

Assim, para gerar os dados necessários para o cálculo do IGI das alternativas avaliadas comparativamente, a valoração dos parâmetros de magnitude e importância está organizada nos Quadros 3, 4, 5 e 6.

Como exemplo, no cenário de manutenção da obra o impacto sobre a perda de biodiversidade é negativo (-), tem duração permanente (3/9) e extensão local (2/9), no entanto sua intensidade é baixa (1/9), por conta das condições prévias ao distúrbio, resultando em uma magnitude de -6/9, como pode ser visto no Quadro 3. Por sua vez, no caso de desfazimento da obra, esse impacto também seria permanente (3/9) e local (2/9), mas positivo (+) e com alta intensidade (3/9), pois permitiria a revegetação em condições melhores que a anterior à obra, totalizando magnitude de 8/9 (Quadro 4).

No primeiro caso, tem-se que a acumulação sobre a perda de biodiversidade é incerta (2/9) e irreversível (3/9) em caso de manutenção da obra, embora com sensibilidade parcial (2/9), em função das condições de urbanização no entorno, totalizando importância valorada em 7/9 para o impacto negativo (Quadro 5). Em contraste, com o desfazimento da obra a acumulação é presente (3/9) e reversível (1/9), mas também parcial (2/9), tendo em vista a urbanização adjacente, alcançando importância de 6/9 para o impacto de natureza positiva, que, nesse cenário, contribuiria para a restituição do hábitat natural (Quadro 6).

De acordo com os valores apresentados, a memória de cálculo do IGI das alternativas avaliadas pode ser consultada no Anexo A, que, pela aplicação da equação 12, resulta em (Equação 15):

$\boldsymbol{I} \boldsymbol{G} \boldsymbol{I}=\delta_{\lambda \tau}\left(\frac{1}{n}\left(\mathbf{M}_{\lambda \tau} \cdot\left[\mathbf{I}_{\lambda \tau}\right]^{\mathrm{T}}\right)\right)=\left[i g i_{\lambda 1} i g i_{\lambda 2}\right]=\left[i g i_{m} i g i_{d}\right]=[0,12-0,01]$

Logo, com a equação 13, tem-se que a melhor alternativa de projeto para a regularização do caso em estudo corresponde a (Equação 16):

$A_{\lambda^{\prime} \tau^{\prime}}=\max (\boldsymbol{I G I})=\left[i g i_{m}\right]=[0,12]=A_{m}$

Assim, nos termos do Decreto federal n. ${ }^{\circ} 6.514$ de 2008, recomenda-se que a regularização da obra não implique o seu desfazimento, pois o cenário de manutenção apresentou o melhor IGI. 
Quadro 3 - Análise da magnitude dos impactos para o cenário de manutenção $\left(A_{\mathrm{m}}\right)$.

\begin{tabular}{|c|c|c|c|c|c|c|c|c|c|c|}
\hline$P_{j}$ & $m_{k j}$ & $w_{k j}$ & $i_{1}$ & $i_{2}$ & $i_{3}$ & $i_{4}$ & $i_{5}$ & $i_{6}$ & $i_{7}$ & $i_{8}$ \\
\hline $\begin{array}{l}\frac{\overline{0}}{0} \\
\frac{0}{0} \\
\frac{0}{0} \\
\overline{0} \\
\square\end{array}$ & 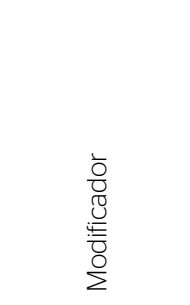 & 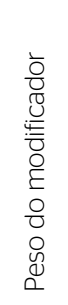 & 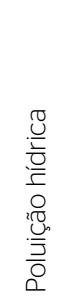 & 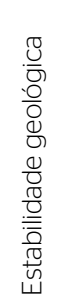 & 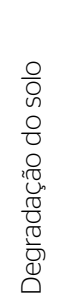 & $\begin{array}{l}\frac{0}{0} \\
\frac{\pi}{0} \\
\frac{0}{0} \\
\frac{0}{0} \\
\frac{2}{0} \\
\frac{0}{0} \\
\frac{0}{0} \\
\frac{\pi}{0} \\
\frac{0}{0}\end{array}$ & 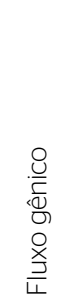 & 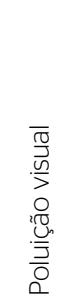 & 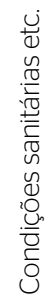 & 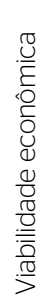 \\
\hline \multirow[b]{2}{*}{ Duração $\left(p_{1}\right)$} & Curta a média & $1 / 9$ & 0 & 0 & 0 & 0 & 0 & 0 & 0 & 0 \\
\hline & Longo prazo & $2 / 9$ & 0 & 0 & 0 & 0 & 0 & 0 & 0 & 0 \\
\hline Extensão $\left(p_{2}\right)$ & Regional & $3 / 9$ & +1 & 0 & 0 & 0 & 0 & 0 & 0 & 0 \\
\hline \multirow{3}{*}{ Intensidade $\left(p_{3}\right)$} & Baixa & $1 / 9$ & +1 & 0 & 0 & -1 & -1 & -1 & $\mathrm{O}$ & 0 \\
\hline & Média & $2 / 9$ & $\mathrm{O}$ & O & +1 & O & $\mathrm{O}$ & 0 & O & +1 \\
\hline & Alta & $3 / 9$ & 0 & +1 & 0 & 0 & 0 & 0 & +1 & 0 \\
\hline \multicolumn{2}{|l|}{$M_{\lambda 1}=M_{m}=\left[\mu_{1} \mu_{2} \ldots \mu_{n}\right]$} & $\mu_{i}$ & $7 / 9^{*}$ & $8 / 9$ & $7 / 9$ & $-6 / 9$ & $-6 / 9$ & $-6 / 9$ & $8 / 9$ & $7 / 9$ \\
\hline
\end{tabular}

*Memorial de cálculo: $\mu=1$ (1/9.0+2/9.0+3/9.1+1/9.0+2/9.0+3/9.0+1/9.1+2/9.0+3/9.0) = 7/9

Quadro 4 - Análise da magnitude dos impactos para o cenário de desfazimento $\left(A_{\mathrm{d}}\right)$.

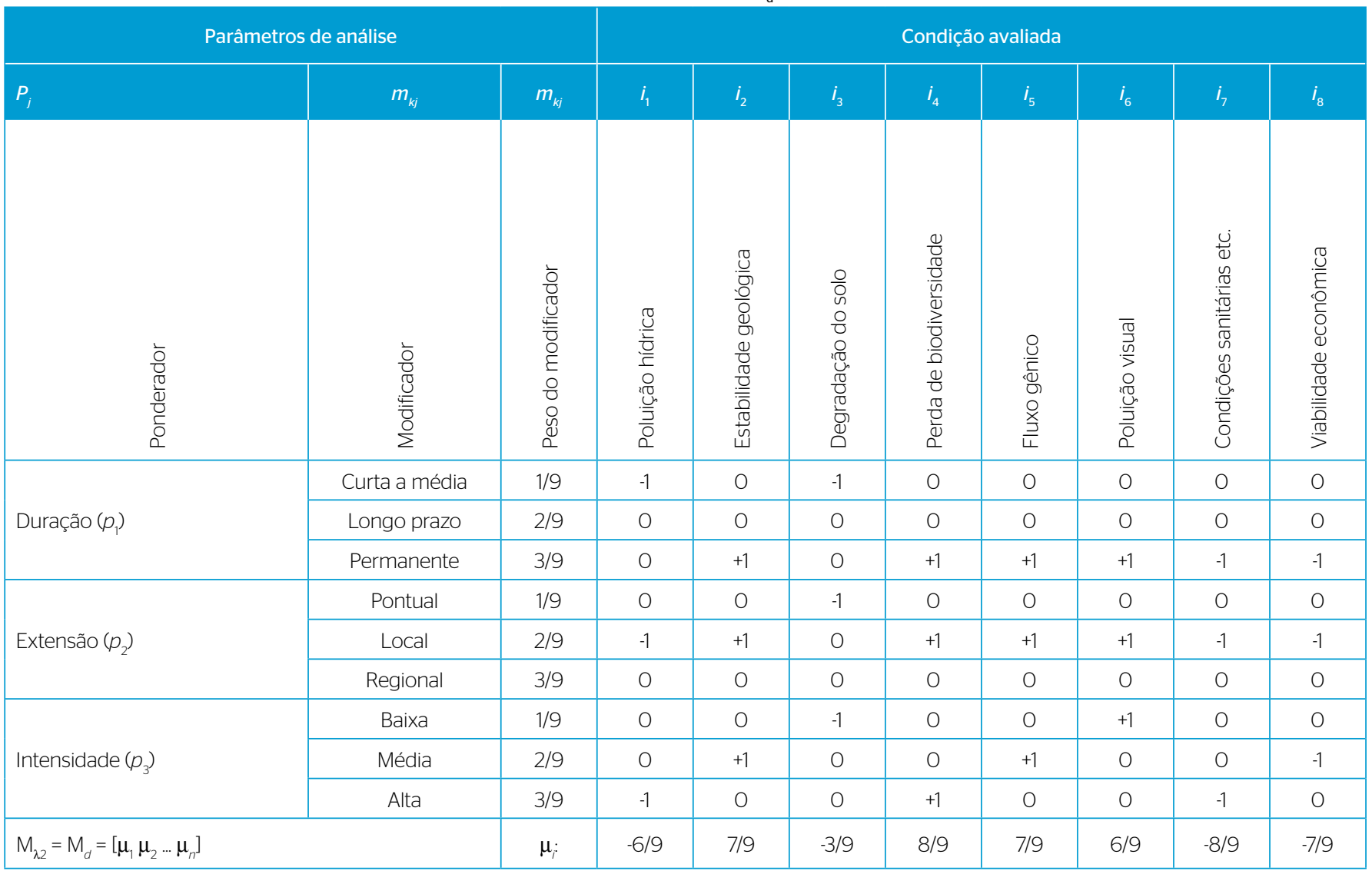


Quadro 5 - Análise da importância dos impactos para o cenário de manutenção $\left(A_{\mathrm{m}}\right)$.

\begin{tabular}{|c|c|c|c|c|c|c|c|c|c|c|}
\hline \multicolumn{3}{|c|}{ Parâmetros de análise } & \multicolumn{8}{|c|}{ Condição avaliada } \\
\hline$P_{j}$ & $m_{k j}$ & $m_{k j}$ & $i_{1}$ & $i_{2}$ & $i_{3}$ & $i_{4}$ & $i_{5}$ & $i_{6}$ & $i_{7}$ & $i_{8}$ \\
\hline $\begin{array}{l}\overline{0} \\
\frac{0}{0} \\
\frac{0}{0} \\
\frac{0}{0} \\
\overline{0} \\
0\end{array}$ & 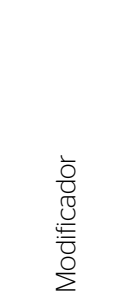 & 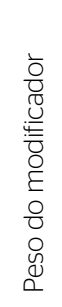 & 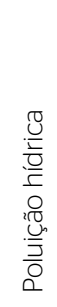 & 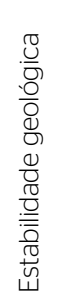 & 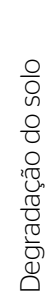 & $\begin{array}{l}\frac{0}{0} \\
\frac{\pi}{0} \\
\frac{0}{0} \\
\frac{\pi}{10} \\
\frac{0}{0} \\
\frac{0}{0} \\
\frac{0}{0} \\
\frac{\pi}{0} \\
\frac{\pi}{0} \\
\frac{0}{0}\end{array}$ & 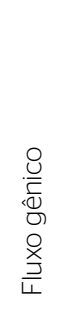 & 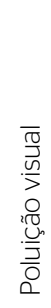 & 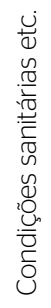 & 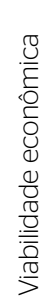 \\
\hline \multirow{3}{*}{ Acumulação $\left(p_{4}\right)$} & Ausente & $\mathrm{O}$ & 1 & 1 & 0 & O & 1 & 0 & 0 & O \\
\hline & Incerta & $2 / 9$ & $\mathrm{O}$ & $\mathrm{O}$ & 1 & 1 & O & 1 & 1 & 1 \\
\hline & Presente & $3 / 9$ & $\mathrm{O}$ & $\mathrm{O}$ & 0 & $\mathrm{O}$ & $\mathrm{O}$ & O & $\mathrm{O}$ & $\mathrm{O}$ \\
\hline \multirow{3}{*}{ Reversibilidade $\left(p_{5}\right)$} & $\operatorname{Sim}$ & $1 / 9$ & 1 & 1 & $\mathrm{O}$ & $\mathrm{O}$ & O & $\mathrm{O}$ & O & $\mathrm{O}$ \\
\hline & Talvez & $2 / 9$ & $\mathrm{O}$ & $\mathrm{O}$ & $\mathrm{O}$ & $\mathrm{O}$ & $\mathrm{O}$ & $\mathrm{O}$ & 1 & $\mathrm{O}$ \\
\hline & Não & $3 / 9$ & 0 & $\mathrm{O}$ & 1 & 1 & 1 & 1 & O & 1 \\
\hline \multirow{3}{*}{ Sensibilidade $\left(p_{6}\right)$} & Nula & $\mathrm{O}$ & $\mathrm{O}$ & 1 & $\mathrm{O}$ & $\mathrm{O}$ & $\mathrm{O}$ & $\mathrm{O}$ & $\mathrm{O}$ & $\mathrm{O}$ \\
\hline & Parcial & $2 / 9$ & 1 & O & 1 & 1 & 1 & 1 & O & $\mathrm{O}$ \\
\hline & Total & $3 / 9$ & 0 & 0 & 0 & 0 & 0 & 0 & 1 & 1 \\
\hline \multicolumn{2}{|l|}{$I_{\lambda 1}=I_{m}=\left[i_{1} i_{2} \ldots i_{n}\right]$} & $i_{i}$ & $3 / 9$ & $1 / 9$ & $7 / 9$ & $7 / 9$ & $5 / 9$ & $7 / 9$ & $7 / 9$ & $8 / 9$ \\
\hline
\end{tabular}

Quadro 6 - Análise da importância dos impactos para o cenário de desfazimento $\left(A_{\mathrm{d}}\right)$.

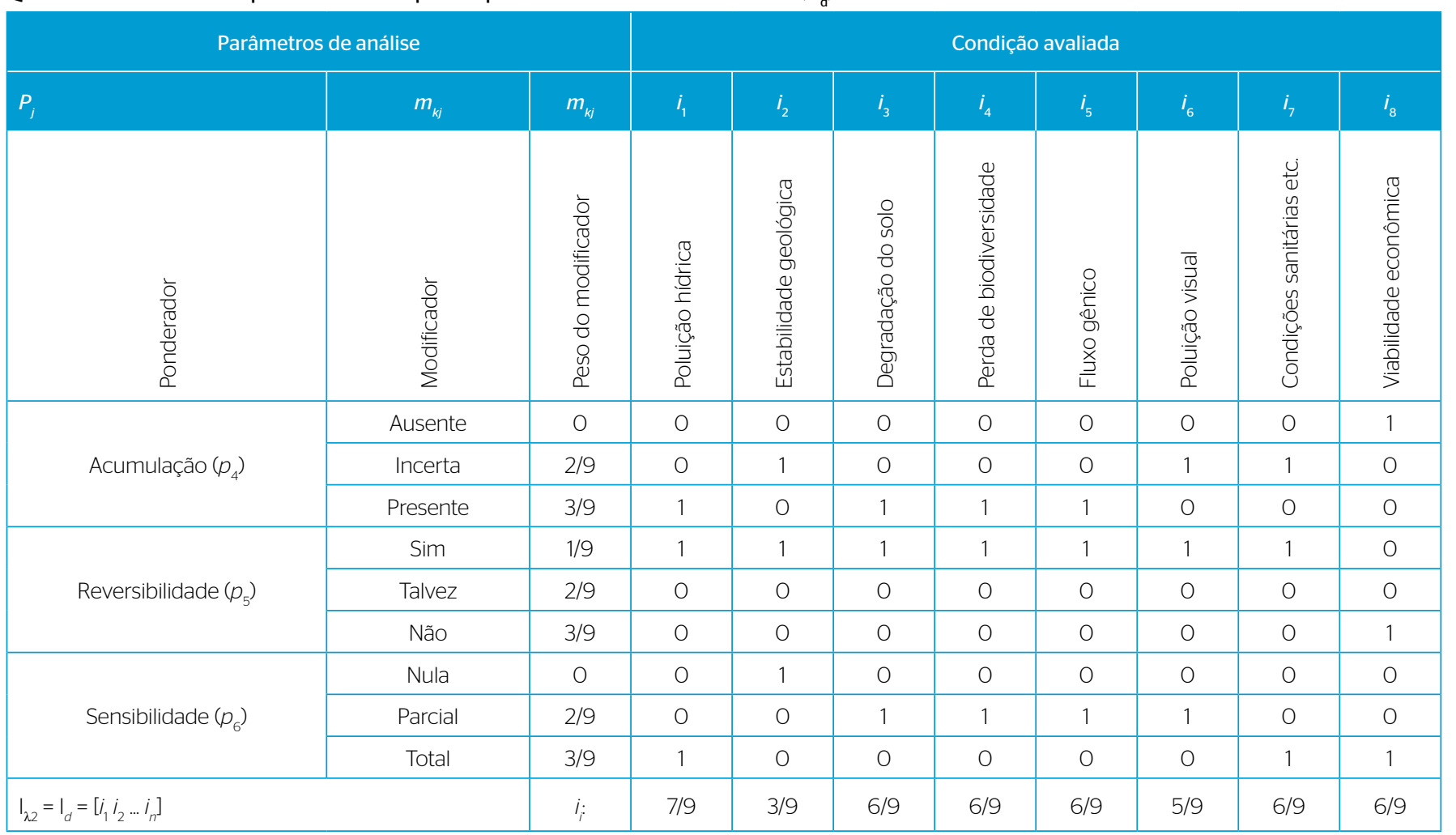




\section{CONCLUSÕES}

No que se refere à análise ambiental comparativa para fins de adequação de obras irregulares, com base nos resultados, pôde-se concluir que o procedimento proposto representa uma contribuição metodológica adequada para subsidiar tecnicamente, de forma clara e objetiva, decisões relacionadas à manutenção ou ao desfazimento dessas obras.

O método proposto contribui para a padronização de avaliações ambientais para tomada de decisões, especialmente para empreendimentos de pequeno e médio portes, os quais muitas vezes são avaliados de acordo com atributos unicamente qualitativos, levando em consideração o consenso dos atores envolvidos. O método proposto não elimina a subjetividade da avaliação, contudo introduz um procedimento lógico e organizado que subsidia a tomada de decisão baseada em parâmetros quantitativos.

Como considerações finais, salienta-se que a importância da preservação ambiental constitui imperiosa premissa para a melhoria da qualidade de vida e para a conservação dos recursos naturais, logo, vital para o alcance de cidades saudáveis e sustentáveis.
Todavia, o equilíbrio ecológico deve pautar-se na adequada conciliação entre a preservação do meio natural e a viabilidade do meio construído, igualmente fundamentais para o bem-estar, a saúde e a segurança das populações humanas.

Com base no exposto, este trabalho revela a importância da análise caso a caso com o uso de procedimentos fundamentados e criteriosos, para que a generalização, não raro enraizada em preconceitos desprovidos de fundamentação técnica, não constitua impactos igualmente nefastos à sustentabilidade ambiental, isto é, aos pilares que sustentam a justiça social, a viabilidade econômica e a conservação da natureza.

Então, uma vez comprovado o maior prejuízo ambiental do desfazimento de uma determinada obra irregular em comparação aos causados pela sua manutenção, tal como no estudo de caso em análise, não é cabível a exigibilidade de sua desconstrução; entretanto, a manutenção da obra de ser condicionada à implantação de medidas mitigadoras e, quando for o caso, de ações de compensação ambiental.

\section{REFERÊNCIAS}

ANDRADE, M.R.M.(1999) Cartografia deaptidão paraassentamentos urbanos do município de Guarulhos. 154 f. Dissertação (Mestrado em Geografia) - Universidade de São Paulo, São Paulo.

BRASIL. (1986) Resolução Conama n.o 001, de 23 de janeiro de 1986. Dispõe sobre Estudo de Impacto Ambiental. Disponível em: <http://www.mma.gov.br/port/conama/res/res86/res0186.html>. Acesso em: 1.॰ abr. 2014

BRASIL. (2008) Decreto Federal n.o 6.514 de 2008. Dispõe sobre infrações e sanções administrativas ao meio ambiente, estabelece o processo administrativo federal para apuração destas infrações, e dá outras providências. Disponivel em: <http://www.planalto.gov.br/ccivil_03/_ ato2007-2010/2008/decreto/d6514.htm>. Acesso em: 1.0 abr. 2014

BROWN, M.T.; ULGIATI, S. (1997) Emergy-based indices and ratios to evaluate sustainability: monitoring economies and technology toward environmentally sound innovation. Ecological Engineering, v. 9, n. 1-2, p. 51-69

COMISSÃO MUNDIAL SOBRE O MEIO AMBIENTE E DESENVOLVIMENTO. (1991) NosSo futuro comum. Rio de Janeiro: FGV. 430 p.

COUTINHO, J.M.V.C. (1984) Carta geológica da região metropolitana de São Paulo. Escala 1:50.000. São Paulo: Emplasa.

FISCHER, D.W:; DAVIES, G.S. (1973) An approach to assessing environmental impacts. Journal of Environmental Management, v. 1. p. 207-227.
GUARULHOS ONLINE. (2010) Fauna de Guarulhos tem cerca de 500 animais silvestres. Disponível em: <http://www.guarulhosonline. com/news/sociedade/fauna-de-guarulhos-tem-cerca-de-500animais-silvestres/>. Acesso em: 1 abr. 2014

INSTITUTO FLORESTAL - IF. (2005) Inventário Florestal da Vegetação Natural do Estado de São Paulo. São Paulo: Imprensa Oficial. 200 p.

INSTITUTO DE PESQUISAS TECNOLÓGICAS - IPT. (1981) Mapa geomorfológico do Estado de São Paulo. Escala 1: 500.000. São Paulo: IPT.

KHANNA, N. (2000) Measuring environmental quality: an index of pollution. Ecological Economics, v. 35, n. 2, p. 191-202.

LEOPOLD, L.B.; CLARKE, F.S.; HANSHAW, B. (1971) A procedure for evaluating environmental impact. Washington: U. S. Geological Survey. 13 p.

MITCHELL, G. (1996) Problems and fundamentals of sustainable development indicators. Sustainable Development, v. 4, n. 1, p. 1-11.

OLIVEIRA, F.C.; MOURA, H.J.T. (2009) Uso das metodologias de avaliação de impacto ambiental em estudos realizados no Ceará Pretexto, v.10, n. 4, p. 79-98.

PIRES NETO, A.G. (2004) Tipos de relevos e compartimentos morfoestruturais das folhas de Guarulhos e Itaquaquecetuba (SP) Guarulhos: UnG. 17 p. 
PREFEITURA MUNICIPAL DE GUARULHOS. (2004a) DecretoLei municipal n.o 6.055 de 2004. Dispõe sobre o plano diretor do município de Guarulhos (SP) Disponível em: <http://leis. guarulhos.sp.gov.br/06_prefeitura/leis/leis_download/06055lei. pdf>. Acesso em: 1.0 abr. 2014.

PREFEITURA MUNICIPAL DE GUARULHOS. (2004b) Plano diretor de drenagem de Guarulhos (SP) Disponível em: $<$ http://servicos.guarulhos.sp.gov.br/destaques/coord_ assunt_aerop/plano_diretor_drenagem_07.pdf>. Acesso em: 10 out. 2012

GOVERNO DO ESTADO DE SÃO PAULO. (2007) Mapa de conectividade do Estado deSão Paulo.São Paulo: Fapesp. Disponível em: <http://www.ambiente.sp.gov.br/sifesp/conectividade/>. Acesso em: 1. ${ }^{\circ}$ abr. 2014.

RUIZ, J.A. (1996) Metodologia científica: guia para eficiência nos estudos. 4. ed. São Paulo: Atlas. 184 p.
SAMUEL-JOHNSON, K.; ESTY, D.C. (2000) Pilot environmental sustainability index report. Davos (Switzerland): World Economic Forum: Annual Meeting. 41 p. Disponível em: <http://sedac.ciesin. org/es/esi/ESI_OO.pdf>. Acesso em: 1.0 abr. 2014.

SÁNCHEZ, L.E. (2006) Avaliação de impacto ambiental: conceitos e métodos. São Paulo: Oficina de Textos. 496 p.

SANTOS, F.S. (2004) Planejamento ambiental: teoria e prática. São Paulo: Oficina de Textos. 183 p.

SICHE, R.; AGOSTINHO, F; ORTEGA, E.; ROMEIRO, A. (2007) Índices versus indicadores: precisões conceituais na discussão da sustentabilidade de países. Ambiente \& Sociedade, v. 10, n. 2, p. 137-148.

TOMMASI, L.R. (1993) Estudo de impacto ambiental.São Paulo: Cetesb.354 p.

WACKERNAGEL, M.; REES, W. (1996) Our ecological footprint: reducing human impact on the Earth. Filadélfia, PA: New Society Publishers. 160 p. 


\section{ANEXO A}

Memória de cálculo do índice global de impacto para a manutenção da obra

\section{Poluição hídrica}

$\mu_{1}=3 / 9+3 / 9+1 / 9=7 / 9$

$\mathbf{u}_{1}=0+1 / 9+2 / 9=3 / 9$

$\sigma_{1}=7 / 9 * 3 / 9=7 / 27$

Estabilidade geológica

$\mu_{2}=3 / 9+2 / 9+3 / 9=8 / 9$

$\mathrm{r}_{2}=\mathrm{O}+1 / 9+\mathrm{O}=1 / 9$

$\sigma_{2}=8 / 9 * 1 / 9=8 / 81$

Degradação do solo

$\mu_{3}=3 / 9+2 / 9+2 / 9=7 / 9$

$\mathrm{r}_{3}=2 / 9+3 / 9+2 / 9=7 / 9$

$\sigma_{3}=7 / 9 * 7 / 9=49 / 81$

Perda de biodiversidade

$\mu_{4}=-3 / 9-2 / 9-1 / 9=-6 / 9$

$\mathbf{u}_{4}=2 / 9+3 / 9+2 / 9=7 / 9$

$\sigma_{4}=-6 / 9 * 7 / 9=-14 / 27$

\section{Fluxo gênico}

$\mu_{5}=-3 / 9-2 / 9-1 / 9=-6 / 9$

$\mathbf{1}_{5}=0+3 / 9+2 / 9=5 / 9$

$\sigma_{5}=-6 / 9 * 5 / 9=-10 / 27$

Poluição visual

$\mu_{6}=-3 / 9-2 / 9-1 / 9=-6 / 9$

$u_{6}=2 / 9+3 / 9+2 / 9=7 / 9$

$\sigma_{6}=-6 / 9 * 7 / 9=-14 / 27$

\section{Condições sanitárias}

$\mu_{7}=3 / 9+2 / 9+3 / 9=8 / 9$

$\mathrm{l}_{7}=2 / 9+2 / 9+3 / 9=7 / 9$

$\sigma_{7}=8 / 9 * 7 / 9=56 / 81$

\section{Viabilidade econômica}

$\mu_{8}=3 / 9+2 / 9+2 / 9=7 / 9$

$\mathbf{u}_{8}=2 / 9+3 / 9+3 / 9=8 / 9$

$\sigma_{8}=7 / 9 * 8 / 9=56 / 81$

Índice global de impacto

igi $=1 / 8(7 / 27+8 / 81+49 / 81-14 / 27-10 / 27-14 / 27+56 / 81+56 / 81)$

igi $=0,117$
Memória de cálculo do índice global de impacto para o desfazimento da obra

Poluição hídrica

$\mu_{1}=-1 / 9-2 / 9-3 / 9=-6 / 9$

$\mathbf{u}_{1}=3 / 9+1 / 9+3 / 9=7 / 9$

$\sigma_{1}=-6 / 9 * 7 / 9=-14 / 27$

\section{Estabilidade geológica}

$\mu_{2}=3 / 9+2 / 9+2 / 9=7 / 9$

$\mathbf{l}_{2}=2 / 9+1 / 9+0=3 / 9$

$\sigma_{2}=7 / 9 * 3 / 9=7 / 27$

\section{Degradação do solo}

$\mu_{3}=-1 / 9-1 / 9-1 / 9=-3 / 9$

$\mathbf{l}_{3}=3 / 9+1 / 9+2 / 9=6 / 9$

$\sigma_{3}=-3 / 9 * 6 / 9=-2 / 9$

Perda de biodiversidade

$\mu_{4}=3 / 9+2 / 9+3 / 9=8 / 9$

$\mathrm{i}_{4}=3 / 9+1 / 9+2 / 9=6 / 9$

$\sigma_{4}=8 / 9 * 6 / 9=16 / 27$

Fluxo gênico

$\mu_{5}=3 / 9+2 / 9+2 / 9=7 / 9$

$\mathbf{u}_{5}=3 / 9+1 / 9+2 / 9=6 / 9$

$\sigma_{5}=7 / 9 * 6 / 9=14 / 27$

Poluição visual

$\mu_{6}=3 / 9+2 / 9+1 / 9=6 / 9$

$\mathbf{u}_{6}=2 / 9+1 / 9+2 / 9=5 / 9$

$\sigma_{6}=6 / 9 * 5 / 9=10 / 27$

\section{Condições sanitárias}

$\mu_{7}=-3 / 9-2 / 9-3 / 9=-8 / 9$

$\mathbf{u}_{7}=2 / 9+1 / 9+3 / 9=6 / 9$

$\sigma_{7}=-8 / 9 * 6 / 9=-16 / 27$

\section{Viabilidade econômica}

$\mu_{8}=-3 / 9-2 / 9-2 / 9=-7 / 9$

$\mathrm{u}_{8}=0+3 / 9+3 / 9=6 / 9$

$\sigma_{8}=-7 / 9 * 6 / 9=-14 / 27$

Índice global de impacto

igi $=1 / 8(-14 / 27+7 / 27-2 / 9+16 / 27+14 / 27+10 / 27-16 / 27-14 / 27)$

igi $=-0,014$ 